\title{
Design High Precision Intelligent Nonlinear-Based Controller
}

\author{
Atefeh Chahkoutahi, Mohammad Reza MoradiPour, Mohammad Gholami, Sirous \\ Ashja and Mohammad Hossein Rahimi \\ Department of Electrical Engineering, Delvar Branch, Islamic Azad University, \\ Delvar, Iran \\ atefehchahkootahi@gmail.com
}

\begin{abstract}
Computed torque controller (CTC) is a significant nonlinear controller under condition of partly uncertain dynamic parameters of system. This controller is used to control of highly nonlinear systems especially for robot manipulators, because this controller is a robust and stable. Conversely, computed torque controller is used in many applications; it has an important drawback namely; nonlinear equivalent dynamic formulation in uncertain dynamic parameter. The nonlinear equivalent dynamic formulation problem in uncertain system can be solved by using artificial intelligence theorem. However fuzzy logic controller is used to control complicated nonlinear dynamic systems, but it cannot guarantee stability and robustness. In this research parallel fuzzy logic theory is used to compensate the system dynamic uncertainty in computed torque controller.
\end{abstract}

Keywords: Computed torque Controller, Robot manipulator, Nonlinear equivalent part, Fuzzy inference system, Compensator

\section{Introduction}

In modern usage, the word of control has many meanings, this word is usually taken to mean regulate, direct or command. The word feedback plays a vital role in the advance engineering and science. The conceptual frame work in Feed-back theory has developed only since world war II. In the twentieth century, there was a rapid growth in the application of feedback controllers in process industries. According to Ogata, to do the first significant work in three-term or PID controllers which Nicholas Minorsky worked on it by automatic controllers in 1922. In 1934, Stefen Black was invention of the feedback amplifiers to develop the negative feedback amplifier[1]. Negative feedback invited communications engineer Harold Black in 1928 and it occurs when the output is subtracted from the input. Automatic control has played an important role in advance science and engineering and its extreme importance in many industrial applications, i.e., aerospace, mechanical engineering and robotic systems. The first significant work in automatic control was James Watt's centrifugal governor for the speed control in motor engine in eighteenth century [2]. There are several methods for controlling a robot manipulator, which all of them follow two common goals, namely, hardware/software implementation and acceptable performance. However, the mechanical design of robot manipulator is very important to select the best controller but in general two types schemes can be presented, namely, a joint space control schemes and an operation space control schemes [3]. Joint space and operational space control are closed loop controllers which they have been used to provide robustness and rejection of disturbance effect. The main target in joint space controller is design a feedback controller that allows the actual motion $\left(q_{a}(t)\right)$ tracking of the desired motion $\left(q_{d}(t)\right)$. This control problem is classified into two main groups. Firstly, transformation the desired motion $X_{d}(t)$ to joint 
variable $q_{d}(t)$ by inverse kinematics of robot manipulators [4]. The main target in operational space controller is to design a feedback controller to allow the actual end-effector motion $X_{a}(t)$ to track the desired endeffector motion $X_{d}(t)$. This control methodology requires a greater algorithmic complexity and the inverse kinematics used in the feedback control loop. Direct measurement of operational space variables are very expensive that caused to limitation used of this controller in industrial robot manipulators[5]. One of the simplest ways to analysis control of multiple DOF robot manipulators are analyzed each joint separately such as SISO systems and design an independent joint controller for each joint. In this methodology, the coupling effects between the joints are modeled as disturbance inputs. To make this controller, the inputs are modeled as: total velocity/displacement and disturbance. Design a controller with the same formulation and different coefficient, low cost hardware and simple structure controller are some of most important independent-joint space controller advantages. Nonlinear controllers divided into six groups, namely, feedback linearization (computed-torque control), passivity-based control, sliding mode control (variable structure control), artificial intelligence control, Lyapunov-based control and adaptive control [6-7].

Computed torque controller (CTC) is a powerful nonlinear controller which it widely used in control of robot manipulator. It is based on feedback linearization and computes the required arm torques using the nonlinear feedback control law. This controller works very well when all dynamic and physical parameters are known but when the robot manipulator has variation in dynamic parameters, in this situation the controller has no acceptable performance[8]. In practice, most of physical systems (e.g., robot manipulators) parameters are unknown or time variant, therefore, computed torque like controller used to compensate dynamic equation of robot manipulator[9]. Research on computed torque controller is significantly growing on robot manipulator application which has been reported in [10]. Vivas and Mosquera have proposed a predictive functional controller and compare to computed torque controller for tracking response in uncertain environment. However both controllers have been used in feedback linearization, but predictive strategy gives better result as a performance. A computed torque control with non parametric regression models have been presented for a robot arm. This controller also has been problem in uncertain dynamic models. Based on [11] and [12] computed torque controller is a significant nonlinear controller to certain systems which it is based on feedback linearization and computes the required arm torques using the nonlinear feedback control law. When all dynamic and physical parameters are known, computed torque controller works fantastically; practically a large amount of systems have uncertainties, therefore sliding mode controller is one of the best case to solve this challenge.

In recent years, artificial intelligence theory has been used in sliding mode control systems. Neural network, fuzzy logic and neuro-fuzzy are synergically combined with nonlinear classical controller and used in nonlinear, time variant and uncertain plant (e.g., robot manipulator). Fuzzy logic controller (FLC) is one of the most important applications of fuzzy logic theory. This controller can be used to control nonlinear, uncertain, and noisy systems. This method is free of some model techniques as in model-based controllers. As mentioned that fuzzy logic application is not only limited to the modelling of nonlinear systems [13] but also this method can help engineers to design a model-free controller. Control robot arm manipulators using model-based controllers are based on manipulator dynamic model. These controllers often have many problems for modelling. Conventional controllers require accurate information of dynamic model of robot manipulator, but most of time these models are MIMO, nonlinear and partly uncertain therefore calculate accurate dynamic model is complicated [13]. The main reasons to use fuzzy logic methodology are able to give approximate recommended solution for uncertain and also certain complicated systems to 
easy understanding and flexible. Fuzzy logic provides a method to design a model-free controller for nonlinear plant with a set of IF-THEN rules [14-17].

Based on mechanical and control methodologies research in robotic system, mechanical design, type of actuators and type of systems drive play important roles to have the best performance controller. This section has focused on the robot manipulator mechanical classification. Types of kinematics chain, i.e., serial Vs. parallel manipulators, and types of connection between link and join actuators, i.e., highly geared systems Vs. direct-drive systems are presented in the following sections because these topics played important roles to select and design the best acceptable performance controllers. A serial link robot is a sequence of joints and links which begins with a base frame and ends with an end-effector. This type of robot manipulators, comparing with the load capacity is more weightily because each link must be supported the weights of all next links and actuators between the present link and end-effector. Serial robot manipulators have been used in automotive industry, medical application, and also in research laboratories. In contrast, parallel robot manipulators design according to close loop which base frame is connected to the end-effector frame with two or more kinematic chains [6]. In the other words, a parallel link robot has two or more branches with some joints and links, which support the load in parallel. Parallel robot have been used in many applications such as expensive flight simulator, medical robotics (i.e., high accuracy, high repeatability, high precision robot surgery), and machinery tools [1]. Parallel links robot manipulators have higher accuracy and faster than serial links robot manipulators but the work space limitation in serial links robot manipulator is lower than parallel links robot manipulator. From control point of view, the coupling between different kinematic chains can generate the uncertainty problems which cause difficult controller design of parallel robot manipulator $[1,6]$. One of the most important classifications in controlling the robot manipulator is how the links have connected to the actuators. This classification divides into two main groups: highly geared (e.g., 200 to 1) and direct drive (e.g., 1 to 1) [1]. High gear ratios reduce the nonlinear coupling dynamic parameters in robot manipulator. In this case, each joint is modeled the same as SISO systems. In high gear robot manipulators which generally are used in industry, the couplings are modeled as a disturbance for SISO systems [13]. Direct drive increases the coupling of nonlinear dynamic parameters of robot manipulators. This effect should be considered in the design of control systems. As a result some control and robotic researchers' works on nonlinear robust controller design [2].

Normal combinations of fuzzy logic methodology (FLM) and computed torque controller (CTC) are to apply fuzzy pzrzllel compensator at the same time [12], while FLM compensates the control error, CTC reduce the error of fuzzy inference system such that the final tracking error is asymptotically stable [11]. The equivalent part is compensating, because CTC and FLM work parallel. In this paper, the asymptotic stability of CTC control with parallel fuzzy logic compensation is proposed (CTC+FLM). The fuzzy inference system is used to approximate the nonlinear plant. A dead one algorithm is applied for the fuzzy control. After the regulation error enter converges to the dead-zone, a super-twisting secondorder computed torque control is used to guarantee finite time convergence of the whole control (FLM+CTC). By means of a Lyapunov approach, we prove that this type of control can ensure finite time convergence and less oscillation than CTC.

This paper is organized as follows; second part focuses on the modeling dynamic formulation based on Lagrange methodology, fuzzy logic methodology and computed torque controller to have a robust control. Third part is focused on the methodology which can be used to reduce the error, increase the performance quality and increase the robustness and stability. Simulation result and discussion is illustrated in forth part which based on trajectory 
following and disturbance rejection. The last part focuses on the conclusion and compare between this method and the other ones.

\section{Theory}

Dynamic Formulation of Robot Manipulator: The equation of a multi degrees of freedom (DOF) robot manipulator is calculated by the following equation[6]:

$$
\boldsymbol{M}(\boldsymbol{q}) \ddot{\boldsymbol{q}}+\boldsymbol{N}(\boldsymbol{q}, \dot{q})=\tau
$$

Where $\tau$ is $n \times 1$ vector of actuation torque, $\mathrm{M}(\mathrm{q})$ is $n \times n$ symmetric and positive define inertia matrix, $N(q, \dot{q})$ is the vector of nonlinearity term, and $\mathrm{q}$ is $n \times 1$ position vector. In equation (1) if vector of nonlinearity term derive as Centrifugal, Coriolis and Gravity terms, as a result robot manipulator dynamic equation can also be written as [6]:

$$
\begin{aligned}
& N(q, \dot{q})=V(q, \dot{q})+G(q) \\
& V(q, \dot{q})=B(q)[\dot{q} \dot{q}]+C(q)[\dot{q}]^{2} \\
& \tau=M(q) \ddot{q}+B(q)[\dot{q} \dot{q}]+C(q)[\dot{q}]^{2}+G(q)
\end{aligned}
$$

Where,

$B(q)$ is matrix of coriolis torques, $C(q)$ is matrix of centrifugal torque, $[\dot{q} \dot{q}]$ is vector of joint velocity that it can give by: $\left[\dot{q}_{1} \cdot \dot{q}_{2}, \dot{q}_{1} \cdot \dot{q}_{3}, \ldots, \dot{q}_{1} \cdot \dot{q}_{n}, \dot{q}_{2} . \dot{q}_{3}, \ldots .\right]^{T}$, and $[\dot{q}]^{2}$ is vector, that it can given by: $\left[\dot{q}_{1}{ }^{2}, \dot{q}_{2}{ }^{2}, \dot{q}_{3}{ }^{2}, \ldots .\right]^{T}$.

In robot manipulator dynamic part the inputs are torques and the outputs are actual displacements, as a result in (4) it can be written as [6];

$$
\ddot{\boldsymbol{q}}=\boldsymbol{M}^{-1}(\boldsymbol{q}) .\{\boldsymbol{\tau}-\boldsymbol{N}(\boldsymbol{q}, \dot{\boldsymbol{q}})\}
$$

To implementation (5) the first step is implement the kinetic energy matrix (M) parameters by used of Lagrange's formulation. The second step is implementing the Coriolis and Centrifugal matrix which they can calculate by partial derivatives of kinetic energy. The last step to implement the dynamic equation of robot manipulator is to find the gravity vector by performing the summation of Lagrange's formulation.

The kinetic energy equation (M) is a $n \times n$ symmetric matrix that can be calculated by the following equation;

$$
\begin{aligned}
& M(\theta)=m_{1} J_{v 1}^{T} J_{v 1}+J_{\omega 1}^{T C 1} I_{1} J_{\omega 1}+m_{2} J_{v 2}^{T} J_{v 2}+J_{\omega 2}^{T C 2} I_{2} J_{\omega 2}+m_{3} J_{v 3}^{T} J_{v 3}+ \\
& J_{\omega 3}^{T C 3} I_{3} J_{\omega 3}+m_{4} J_{v 4}^{T} J_{v 4}+J_{\omega 4}^{T C 4} I_{4} J_{\omega 4}+m_{5} J_{v 5}^{T} J_{v 5}+J_{\omega 5}^{T C 5} I_{5} J_{\omega 5}+m_{6} J_{v 6}^{T} J_{v 6}+ \\
& J_{\omega 6}^{T C 6} I_{6} J_{\omega 6}
\end{aligned}
$$

As mentioned above the kinetic energy matrix in $n$ DOF is a $n \times n$ matrix that can be calculated by the following matrix $[1,6]$

$$
\boldsymbol{M}(\boldsymbol{q})=
$$




$$
\left[\begin{array}{cccccc}
M_{11} & M_{12} & \ldots & \ldots & \ldots . . & M_{1 n} \\
M_{21} & \ldots & \ldots & \ldots & \ldots . & M_{2 n} \\
\ldots & \ldots & \ldots & \ldots & \ldots & \ldots \\
\ldots & \ldots & \ldots & \ldots & \ldots & \ldots \\
\ldots & \ldots & \ldots & \ldots & \ldots & \ldots \\
M_{n .1} & \ldots & \ldots & \ldots & \ldots & M_{n . n}
\end{array}\right]
$$

The Coriolis matrix (B) is a $n \times \frac{n(n-1)}{2}$ matrix which calculated as follows;

$$
\begin{aligned}
& \boldsymbol{B}(\boldsymbol{q})= \\
& {\left[\begin{array}{cccccccccc}
\boldsymbol{b}_{112} & \boldsymbol{b}_{113} & \ldots & \boldsymbol{b}_{11 n} & \boldsymbol{b}_{123} & \ldots & \boldsymbol{b}_{12 n} & \ldots & \ldots & \boldsymbol{b}_{1 . n-1 . n} \\
\boldsymbol{b}_{212} & \ldots & \ldots & \boldsymbol{b}_{21 n} & \boldsymbol{b}_{223} & \ldots & \ldots & \ldots & \ldots & \boldsymbol{b}_{2 . n-1 . n} \\
\ldots & \ldots & \ldots & \ldots & \ldots & \ldots & \ldots & \ldots & \ldots & \ldots \\
\ldots & \ldots & \ldots & \ldots & \ldots & \ldots & \ldots & \ldots & \ldots & \ldots \\
\ldots & \ldots & \ldots & \ldots & \ldots & \ldots & \ldots & \ldots & \ldots & \ldots \\
b_{n .1 .2} & \ldots & \ldots & \boldsymbol{b}_{n .1 . n} & \ldots & \ldots & \ldots & \ldots & \ldots & \boldsymbol{b}_{n . n-1 . n}
\end{array}\right]}
\end{aligned}
$$

and the Centrifugal matrix $(\mathrm{C})$ is a $n \times n$ matrix;

$$
C(q)=\left[\begin{array}{ccc}
C_{11} & \cdots & C_{1 n} \\
\vdots & \ddots & \vdots \\
C_{n 1} & \cdots & C_{n n}
\end{array}\right]
$$

And last the Gravity vector $(\mathrm{G})$ is a $n \times 1$ vector;

$$
G(q)=\left[\begin{array}{c}
g_{1} \\
g_{2} \\
\vdots \\
g_{n}
\end{array}\right]
$$

Figure 1 shows the block diagram of two degrees of freedom robot manipulator.

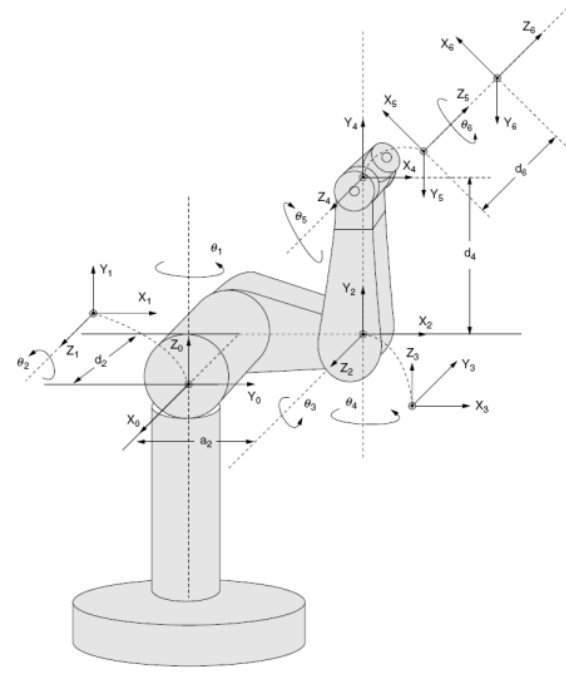

Figure 1. Manipulator Used for Simulations 
Computed Torque Controller: The central idea of Computed torque controller (CTC) is feedback linearization so, originally this algorithm is called feedback linearization controller [3]. It has assumed that the desired motion trajectory for the manipulator $\mathbf{q}_{\mathbf{d}}(\mathbf{t})$, as determined, by a path planner. Defines the tracking error as:

$$
\mathbf{e}(\mathbf{t})=\mathbf{q}_{\mathbf{d}}(\mathbf{t})-\mathbf{q}_{\mathbf{a}}(\mathbf{t})
$$

Where e $(t)$ is error of the plant, $\mathbf{q}_{\mathbf{d}}(\mathbf{t})$ is desired input variable, that in our system is desired displacement, $\mathbf{q}_{\mathbf{a}}(\mathbf{t})$ is actual displacement. If an alternative linear state-space equation in the form $\dot{\mathbf{x}}=\mathbf{A} \mathbf{x}+\mathbf{B U}$ can be defined as

$$
\dot{\mathbf{x}}=\left[\begin{array}{ll}
\mathbf{0} & \mathbf{I} \\
\mathbf{0} & \mathbf{0}
\end{array}\right] \mathbf{x}+\left[\begin{array}{l}
\mathbf{0} \\
\mathbf{I}
\end{array}\right] \mathbf{U}
$$

With $\mathbf{U}=-\mathbf{M}^{-\mathbf{1}}(\mathbf{q}) \cdot \mathbf{N}(\mathbf{q}, \dot{\mathbf{q}})+\mathbf{M}^{-\mathbf{1}}(\mathbf{q}) \cdot \boldsymbol{\tau}$ and this is known as the Brunousky canonical form. By equation (11) and (12) the Brunousky canonical form can be written in terms of the state $\mathbf{x}=\left[\mathbf{e}^{\mathbf{T}} \dot{\mathbf{e}}^{\mathbf{T}}\right]^{\mathbf{T}}$ as $[1]$ :

$$
\frac{\mathbf{d}}{\mathbf{d t}}\left[\begin{array}{l}
\mathbf{e} \\
\dot{\mathbf{e}}
\end{array}\right]=\left[\begin{array}{ll}
\mathbf{0} & \mathbf{I} \\
0 & 0
\end{array}\right] \cdot\left[\begin{array}{l}
\mathbf{e} \\
\dot{\mathbf{e}}
\end{array}\right]+\left[\begin{array}{l}
\mathbf{0} \\
\mathbf{I}
\end{array}\right] \mathbf{U}
$$

With

$$
\mathbf{U}=\ddot{\mathbf{q}}_{\mathbf{d}}+\mathbf{M}^{-1}(\mathbf{q}) \cdot\{\mathbf{N}(\mathbf{q} \cdot \dot{\mathbf{q}})-\tau\}
$$

Then compute the required arm torques using inverse of equation (14), is;

$$
\boldsymbol{\tau}=\mathbf{M}(\mathbf{q})(\ddot{\mathbf{q}} \mathbf{d}-\mathbf{U})+\mathbf{N}(\dot{\mathbf{q}}, \mathbf{q})
$$

This is a nonlinear feedback control law that guarantees tracking of desired trajectory. Selecting proportional-plus-derivative (PD) feedback for $\mathrm{U}(\mathrm{t})$ results in the PD-computed torque controller [6];

$$
\tau=\mathbf{M}(\mathbf{q})\left(\ddot{\mathbf{q}}_{\mathbf{d}}+\mathbf{K}_{\mathbf{v}} \dot{\mathbf{e}}+\mathbf{K}_{\mathbf{p}} \mathbf{e}\right)+\mathbf{N}(\mathbf{q}, \dot{\mathbf{q}})
$$

and the resulting linear error dynamics are

$$
\left(\ddot{\mathbf{q}}_{\mathbf{d}}+\mathbf{K}_{\mathbf{v}} \dot{\mathbf{e}}+\mathbf{K}_{\mathbf{p}} \mathbf{e}\right)=\mathbf{0}
$$

According to the linear system theory, convergence of the tracking error to zero is guaranteed [6]. Where $\mathbf{K}_{\mathbf{p}}$ and $\mathbf{K}_{\mathbf{v}}$ are the controller gains.

Fuzzy Logic Technique: Based on foundation of fuzzy logic methodology; fuzzy logic management has played important rule to design nonlinear management for nonlinear and uncertain systems [5]. However the application area for fuzzy control is really wide, the basic form for all command types of controllers consists of;

Input fuzzification (binary-to-fuzzy $[\mathrm{B} / \mathrm{F}]$ conversion), Fuzzy rule base (knowledge base), Inference engine and Output defuzzification (fuzzy-to-binary [F/B] conversion). The fuzzy inference engine offers a mechanism for transferring the rule base in fuzzy set which it is 
divided into two most important methods, namely, Mamdani method and Sugeno method. Mamdani method is one of the common fuzzy inference systems and he designed one of the first fuzzy managements to control of system engine. Mamdani's fuzzy inference system is divided into four major steps: fuzzification, rule evaluation, aggregation of the rule outputs and defuzzification. Michio Sugeno uses a singleton as a membership function of the rule consequent part. The following definition shows the Mamdani and Sugeno fuzzy rule base [8]

\section{if $x$ is $A$ and $y$ is $B$ then $z$ is $C$ 'mamdani' if $x$ is $A$ and $y$ is $B$ then $z$ is $f(x, y)$ 'sugeno'}

When $x$ and $y$ have crisp values fuzzification calculates the membership degrees for antecedent part. Rule evaluation focuses on fuzzy operation $(A N D / O R)$ in the antecedent of the fuzzy rules. The aggregation is used to calculate the output fuzzy set and several methodologies can be used in fuzzy logic controller aggregation, namely, Max-Min aggregation, Sum-Min aggregation, Max-bounded product, Max-drastic product, Maxbounded sum, Max-algebraic sum and Min-max. Defuzzification is the last step in the fuzzy inference system which it is used to transform fuzzy set to crisp set. Consequently defuzzification's input is the aggregate output and the defuzzification's output is a crisp number. Centre of gravity method $(C O G)$ and Centre of area method $(C O A)$ are two most common defuzzification methods [9].

\section{Methodology}

Computed torque controller (CTC) is an important nonlinear controller in a partly uncertain dynamic system's parameters. This controller is used in several applications such as in robotics, process control, aerospace and power electronics. Computed torque controller is used to control of nonlinear dynamic systems particularly for robot manipulators, because it has a suitable control performance and it is a robust and stable. Conversely pure computed torque controller is a high-quality nonlinear controller; it has an important problem; nonlinear equivalent dynamic formulation in uncertain dynamic parameter. To compensate the equivalent dynamic problems, this research is focused on applied parallel fuzzy logic theorem in computed torque controller as a compensator. Fuzzy logic theory is used in parallel with computed torque controller to compensate the limited uncertainty in system's dynamic. In this method fuzzy logic theorem is applied to computed torque controller to remove the nonlinear uncertainty part which it is based on nonlinear dynamic formulation. To achieve this goal, the dynamic equivalent part of pure computed torque controller is modeled by Mamdani's performance/ error-based fuzzy logic methodology. Another researcher's method is based on applied fuzzy logic theorem in computed torque controller to design a fuzzy model-based controller. This technique was employed to obtain the desired control behavior with a number of information about dynamic model of system and a fuzzy control was applied to reinforce system performance. Equivalent part of computed torque controller is based on nonlinear dynamic formulations of robot manipulator. Robot manipulator's dynamic formulations are highly nonlinear and some of parameters are unknown therefore design a controller based on dynamic formulation is complicated. To solve the challenge of computed torque controller based on nonlinear dynamic formulation this research is focused on compensate the nonlinear equivalent formulation by parallel fuzzy logic controller. In this method; dynamic nonlinear equivalent part is modelled by performance/error-based fuzzy logic controller. In this method; 
error based Mamdani's fuzzy inference system has considered with two inputs, one output and totally 25 rules.

The parallel fuzzy error-based compensator of computed torque controller's output is written;

$$
\hat{\boldsymbol{\tau}}=\tau_{e q_{f u z z y}}+\tau_{C T C}
$$

Based on fuzzy logic methodology

$$
f(x)=U_{f u z z y}=\sum_{l=1}^{M} \theta^{T} \zeta(x)
$$

where $\boldsymbol{\theta}^{\boldsymbol{T}}$ is adjustable parameter (gain updating factor) and $\boldsymbol{\zeta}(\boldsymbol{x})$ is defined by;

$$
\zeta(x)=\frac{\sum_{i} \mu\left(x_{i}\right) x_{i}}{\sum_{i} \mu\left(x_{i}\right)}
$$

Where $\boldsymbol{\mu}\left(\boldsymbol{x}_{\boldsymbol{i}}\right)$ is membership function. $\boldsymbol{\tau}_{\boldsymbol{f u z z} \boldsymbol{y}}$ is defined as follows;

$$
\tau_{f u z z y}=\sum_{l=1}^{M} \theta^{T} \zeta(x)=[(B+C+G)]
$$

As mentioned in Figure 4, design an error-based parallel fuzzy compensate of equivalent part based on Mamdani's fuzzy inference method has four steps, namely, fuzzification, fuzzy rule base and rule evaluation, aggregation of the rule output (fuzzy inference system) and defuzzification.

Fuzzification: the first step in fuzzification is determine inputs and outputs which, it has two inputs $(e, \dot{e})$ and one output $\left(\tau_{f u z z y}\right)$. The inputs are error (e) which measures the difference between desired and actual output position, and the change of error $(\dot{e})$ which measures the difference between desired and actual velocity and output is fuzzy equivalent torque. The second step is chosen an appropriate membership function for inputs and output which, to simplicity in implementation because it is a linear function with regard to acceptable performance triangular membership function is selected in this research. The third step is chosen the correct labels for each fuzzy set which, in this research namely as linguistic variable.

Fuzzy Rule Base and Rule Evaluation: the first step in rule base and evaluation is to provide a least structured method to derive the fuzzy rule base which, expert experience and control engineering knowledge is used because this method is the least structure of the other one and the researcher derivation the fuzzy rule base from the knowledge of system operate and/or the classical controller. Design the rule base of fuzzy inference system can play important role to design the best performance of parallel fuzzy plus computed torque controller, that to calculate the fuzzy rule base the researcher is used to heuristic method which, it is based on the behavior of the control of robot manipulator. Rule evaluation focuses on operation in the antecedent of the fuzzy rules in fuzzy computed torque controller. This part is used $A N D / O R$ fuzzy operation in antecedent part which $A N D$ operation is used.

Aggregation of the Rule Output (Fuzzy inference): based on fuzzy methodology, Max-Min aggregation is used in this work. 
Defuzzification: The last step to design fuzzy inference in our parallel fuzzy compensator plus computed torque controller is defuzzification. This part is used to transform fuzzy set to crisp set, therefore the input for defuzzification is the aggregate output and the output of it is a crisp number. Based on fuzzy methodology Center of gravity method (COG) is used in this research.

\section{Result and Discussion}

In this section, we use a benchmark model, robot manipulator, to evaluate our control algorithms. We compare the following managements: PD computed torque controller and parallel fuzzy inference compensator plus computed torque controller which is proposed method in this paper. The simulation was implemented in MATLAB/SIMULINK environment.

Close Loop Response of Robot Manipulator Trajectory Planning: Figure 2 illustrates the tracking performance in these controllers.
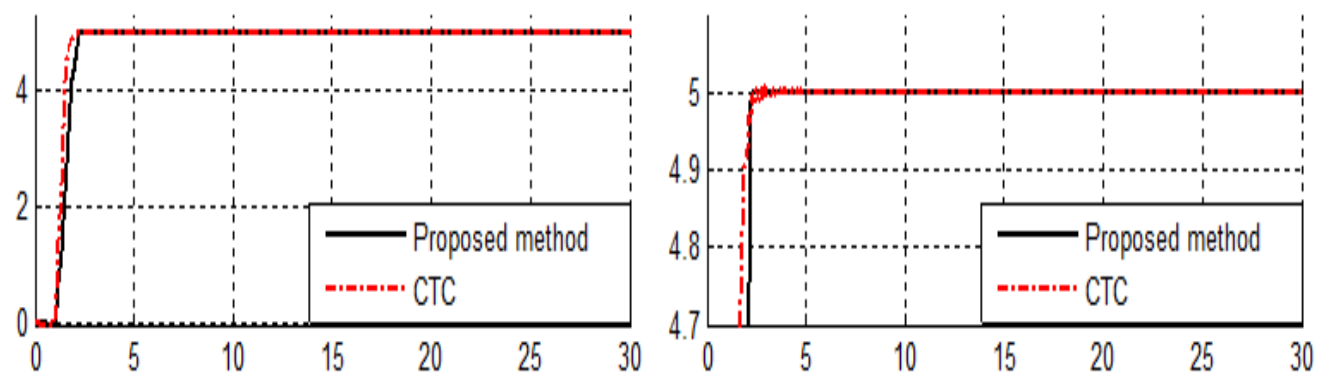

Figure 2. Pure CTC and Proposed Method Trajectory Following without Disturbance

Based on Figure 2; pure PD controller has a slight oscillation in all links, because robot is a highly nonlinear system and control of this system by pure nonlinear method is very difficult.

Close Loop Response of Trajectory Planning in Presence of Disturbance: Figure 3 demonstrates the power disturbance elimination in these controllers in presence of disturbance for robot. The disturbance rejection is used to test the robustness.
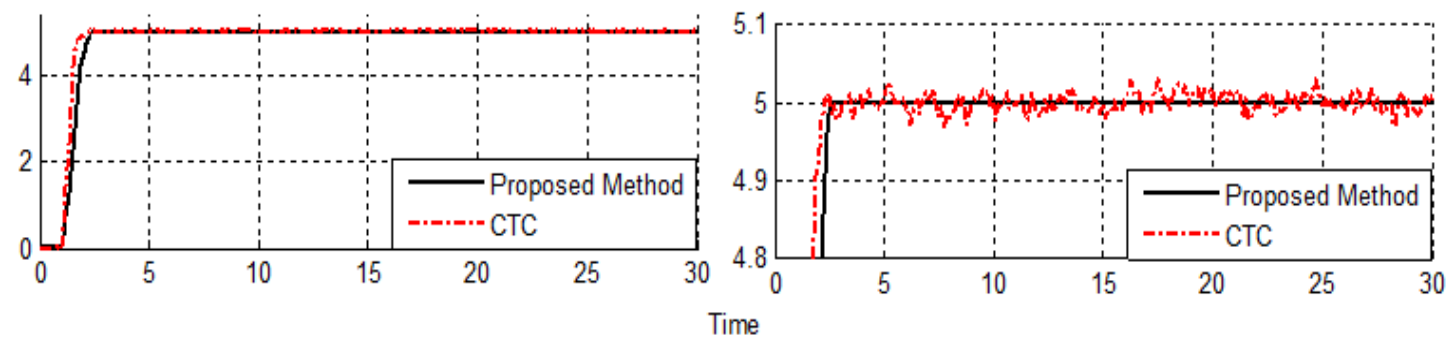

Figure 3. Pure CTC and Proposed Method Trajectory Following with Disturbance 
Based on Figure 3; by comparison between CTC and proposed method, proposed methodology is more stable and robustness than pure nonlinear controller.

\section{Conclusion}

The main contribution of this paper is compensating the nonlinear model base controller by nonlinear artificial intelligence model-free compensator. The structure of computed torque controller with parallel fuzzy inference compensator is new. We propose parallel structure compensator to reduce the error and increase the stability. The key technique is dead-zone, such that fuzzy inference compensator and sliding mode control can be switched automatically. Based on proposed methodology, artificial parallel fuzzy inference system is used to compensate the uncertainty in model-base nonlinear controller.

\section{References}

[1] G. Robinson and J. Davies, "Continuum robots - a state of the art", Proc. IEEE International Conference on Robotics and Automation, Detroit, MI, vol. 4, (1999), pp. 2849-2854.

[2] I. D. Walker, D. Dawson, T. Flash, F. Grasso, R. Hanlon, B. Hochner, W. M. Kier, C. Pagano, C. D. Rahn and Q. Zhang, "Continuum Robot Arms Inspired by Cephalopods, Proceedings SPIE Conference on Unmanned Ground Vehicle Technology VII, Orlando, FL, (2005), pp. 303-314.

[3] K. Suzumori, S. Iikura and H. Tanaka, "Development of Flexible Microactuator and it's Applications to Robotic Mechanisms", Proceedings IEEE International Conference on Robotics and Automation, Sacramento, California, (1991), pp. 1622-1627.

[4] D. Trivedi, C. D. Rahn, W. M. Kier and I. D. Walker, "Soft Robotics: Biological Inspiration, State of the Art, and Future Research", Applied Bionics and Biomechanics, vol. 5, no. 2, (2008), pp. 99-117.

[5] W. McMahan, M. Pritts, V. Chitrakaran, D. Dienno, M. Grissom, B. Jones, M. Csencsits, C. D. Rahn, D. Dawson and I. D. Walker, "Field Trials and Testing of "OCTARM" Continuum Robots", Proc. IEEE International Conference on Robotics and Automation, (2006), pp. 2336-2341.

[6] W. McMahan and I. D. Walker, "Octopus-Inspired Grasp Synergies for Continuum Manipulators", Proc. IEEE International Conference on Robotics and Biomimetics, (2009), pp. 945- 950.

[7] I. Boiko, L. Fridman, A. Pisano and E. Usai, "Analysis of chattering in systems with second-order sliding modes", IEEE Transactions on Automatic Control, vol. 52, no. 11, (2007), pp. 2085-2102.

[8] J. Wang, A. Rad and P. Chan, "Indirect adaptive fuzzy sliding mode control: Part I: fuzzy switching", Fuzzy Sets and Systems, no. 1, vol. 122, (2001), pp. 21-30.

[9] J. J. E. Slotine, "Sliding controller design for non-linear systems", International Journal of Control, no. 2, vol. 40, (1984), pp. 421-434.

[10] R. Palm, "Sliding mode fuzzy control", IEEE conference proceeding, (2002), pp. 519-526.

[11] H. Elmali and N. Olgac, "Implementation of sliding mode control with perturbation estimation (SMCPE)", Control Systems Technology, IEEE Transactions on, vol. 4, no. 1, (2002), pp. 79-85.

[12] J. Moura and N. Olgac, "A comparative study on simulations vs. experiments of SMCPE", IEEE conference proceeding, (2002), pp. 996-1000.

[13] Y. Li and Q. Xu, "Adaptive Sliding Mode Control With Perturbation Estimation and PID Sliding Surface for Motion Tracking of a Piezo-Driven Micromanipulator", Control Systems Technology, IEEE Transactions on, no. 4, vol. 18, (2010), pp. 798-810.

[14] F. Piltan, S. Emamzadeh, Z. Hivand, F. Shahriyari and M. Mirazaei, "PUMA-560 Robot Manipulator Position Sliding Mode Control Methods Using MATLAB/SIMULINK and Their Integration into Graduate/Undergraduate Nonlinear Control, Robotics and MATLAB Courses", International Journal of Robotics and Automation, vol. 3, no. 3, (2012), pp. 106-150.

[15] F. Piltan, M. H. Yarmahmoudi, M. Shamsodini, E. Mazlomian and A. Hosainpour, "PUMA-560 Robot Manipulator Position Computed Torque Control Methods Using MATLAB/SIMULINK and Their Integration into Graduate Nonlinear Control and MATLAB Courses", International Journal of Robotics and Automation, vol. 3, no. 3, (2012), pp. 167-191.

[16] F. Piltan, M. Eram, M. Taghavi, O. R. Sadrnia and M. Jafari, "Nonlinear Fuzzy Model-base Technique to Compensate Highly Nonlinear Continuum Robot Manipulator", IJISA, vol. 5, no. 12, (2013), pp. 135-148,.

[17] F. Piltan, N. Sulaiman, A. Zargari, M. Keshavarz and A. Badri, "Design PID-Like Fuzzy Controller with Minimum Rule Base and Mathematical Proposed On-line Tunable Gain: Applied to Robot Manipulator", International Journal of Artificial Intelligence and Expert System, vol. 2 , no. 4, (2011), pp. 184-195. 